Sociologie et sociétés

SOCIOLOGIE

ETSOCIÉTÉS

\title{
La fugacité des chantiers
}

Inspection du travail et prévention des risques professionnels

dans le secteur du Bâtiment et travaux publics

\section{The Transiency of Construction Sites}

\author{
Work Inspection and the Prevention of Occupational Risk in
} the Construction and Public Works Sector

\section{Nicolas DODIER}

Volume 18, numéro 2, octobre 1986

Travail, santé, prévention

URI : https://id.erudit.org/iderudit/001187ar

DOI : https://doi.org/10.7202/001187ar

Aller au sommaire du numéro

\section{Éditeur(s)}

Les Presses de l'Université de Montréal

\section{ISSN}

0038-030X (imprimé)

1492-1375 (numérique)

Découvrir la revue

Citer cet article

DODIER, N. (1986). La fugacité des chantiers : inspection du travail et prévention des risques professionnels dans le secteur du Bâtiment et travaux publics. Sociologie et sociétés, 18(2), 61-72. https://doi.org/10.7202/001187ar

\section{Résumé de l'article}

Le secteur du bâtiment est caractérisé en France par un taux d'accidents du travail particulièrement élevé. À partir d'une série d'observations de type ethnographique, l'auteur analyse les visites des inspecteurs du travail sur les chantiers comme des processus de construction sociale des risques et comme des négociations autour des dispositifs de sécurité. L'exemple du bâtiment met en évidence l'influence du degré de stabilité des formes d'organisation du travail sur la mise en œuvre d'une politique de prévention des risques professionnels. L'auteur montre en effet les multiples difficultés que la forte instabilité des chantiers crée pour les interventions des inspecteurs, tant dans le repérage des risques que dans le contrôle de l'installation effective des mesures de protection. 


\section{La fugacité des chantiers: Inspection du travail et prévention des risques professionnels dans le secteur du bâtiment et des travaux publics*}

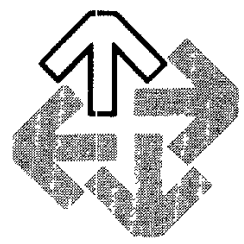

NICOLAS DODIER

Au palmarès des risques d'accidents, les statistiques de la sécurité sociale placent le secteur du bâtiment et travaux publics à la deuxième place, après celui des mines. En France, on comptait 152 accidents du travail pour 1000 salariés dans ce secteur en 1982, alors que dans le vêtement, ou les cuirs et peaux, on en comptait moins de $50 \% 0^{\prime}$. Une définition statistique du risque d'accidents qualifie donc le bâtiment de cible privilégiée pour les actions de prévention des risques professionnels, notamment celles menées par l'inspection du travail. L'inspecteur du travail est en France l'un des multiples acteurs chargés de la sécurité au travail ${ }^{2}$. L'une des missions des inspecteurs du travail est de faire respecter l'application du Code du travail, notamment en matière d'hygiènesécurité. Pour cela, ils disposent d'une arme: le relevé des infractions par procès-verbal. Transmis au Parquet, les procès-verbaux peuvent conduire l'employeur à comparaitre devant le Tribunal correctionnel, et à encourir des amendes ${ }^{3}$.

Les inspecteurs se déplacent sur les chantiers afin d'effectuer des contrôles de sécurité. Ils pointent des situations à risques, ils précisent, parmi les différentes entreprises intervenantes, quels sont les responsables des points problématiques, ils discutent des dispositifs de sécurité à mettre en place, en usant parfois de la menace de procès-verbal pour faire aboutir leurs exigences. Les visites de chantiers sont des suites de scènes entre l'inspecteur et ses interlocuteurs, au cours desquelles sont imposées ou négociées des mesures préventives destinées à remédier aux situations à risques. Avec les inspecteurs entrent sur les chantiers les principes de sécurité. Observer les visites de chantier, c'est donc saisir dans leur déroulement les opérations nécessaires aux acteurs pour définir des dispositifs qui lient ensemble les contraintes de sécurité et les impératifs de la construction des bâtiments. Ces dispositifs sont des objets (garde-corps, échelles), des procédés (adopler un procédé d'élingage correct), des consignes (porter les casques de sécurité), des personnes

* Cet article est tiré d'une recherche plus vaste sur la place de l'hygiène-sécurité dans la pratique professionnelle des inspecteurs et contrôleurs du travail en France. L'étude a bénéficié du financement conjoint du Centre d'Études de l'emploí et du programme mobilisateur technologie, emploi, travail au ministère de la Recherche et de l'Enseignement supérieur (voir N. Dodier, 1986).

1. Ministère du Travail, de l'Emploi et de la Formation professionnelle: «Bilan en 1984 des conditions de travail».

2. Avec les médecins du travail, les ingénieurs de sécurité dans les entreprises ainsi que les contrôleurs de sécurité et les ingénieurs-conseils des services de prévention des caisses régionales d'assurance-maladie.

3. En 1981, les services d'inspection du travail ont dressé 26479 procès-verbaux (dont 9595 en hygiène-sécurité) 16395 amendes ont été infligées (dont 6087 en hygiène-sécurité). Sources: Revue pratique de droit social, n" 467 de mars 1984 , et $n^{\circ} 431$ de mars 1981 ). 
(prévoir quelqu'un pour régler la circulation), etc. L'intrusion de la sécurité dans les activités de travail rencontre des résistances multiples: coûts financiers, contraintes de temps, habitudes de travail, nature des relations de travail dans l'entreprise. Pour les employeurs, répondre aux demandes des inspecteurs, c'est consentir à des investissements dans la sécurité: disposer des objets, procéder à des vérifications (sur les appareils de levage par exemple), établir des consignes, prendre des sanctions si l'ouvrier ne les respecte pas.

Un chantier change sans cesse. À mesure qu'un immeuble est construit, qu'une façade est rénovée, qu'un bâtiment est démoli, la configuration des zones de travail évolue. Les ouvriers ne travaillent plus au même endroit, du béton humide supplante une assise stable et sèche, les platesformes, tout aussi provisoires, sont à nouveau installées. Les statistiques montrent que les chantiers sont dangereux. Mais surtout, les chantiers sont instables. L'objet d'étude de ce texte est la rencontre entre une pratique professionnelle, celle des inspecteurs du travail, et cette fugacité. Les chantiers du bâtiment, parce qu'ils représentent un cas extrême d'évolutions incessantes, sont particulièrement riches pour comprendre comment le degré de stabilité des formes d'organisation du travail ${ }^{4}$ influence la greffe de la sécurité dans les activités de travail.

\section{L'ENQUÊTE}

Ce texte est tiré d'une enquête de terrain menée en 1985 dans trois sections d'inspection du travail. Une section est composée d'un secrétariat, d'un inspecteur du travail et de deux contrôleurs. Chaque section intervient sur un secteur géographique d'un Département, placé quant à lui sous l'autorité d'un directeur départemental du travail. Les inspecteurs s'occupent plus spécialement des entreprises de plus de 50 salariés et les contrôleurs des autres. Sur les chantiers, inspecteurs et contrôleurs ont des pratiques très voisines, nous ne les différencierons pas dans la suite du texte. Le travail de terrain a duré quatre mois. Il consistait à accompagner les inspecteurs dans leur travail quotidien: visites d'établissements, permanences au bureau de la section, participation aux Comités d'hygiène, de sécurité et conditions de travail ${ }^{5}$, enquêtes sur les accidents du travail. En dehors de l'hygiène-sécurité, les inspecteurs ont bien d'autres tâches: décision administrative en cas de licenciements de délégués, fonction d'arbitrage en cas de conflits sociaux, respect de la réglementation du travail dans son ensemble. Après une phase consacrée au tout-venant du travail quotidien, je me suis centré sur les interventions en hygiène-sécurité. Pendant l'enquête, le bâtiment avait été officiellement désigné comme domaine d'actions prioritaires pour la sécurité, en raison d'un taux d'accidents particulièrement élevé. Cette action prioritaire a permis de recueillir un ensemble appréciable d'observations sur les visites de chantier $(n=25$ en tout $)$.

Le déroulement des visites varie du tout au tout avec le type de chantier: depuis la visite d'une journée sur un chantier couvrant toute une zone d'immeubles en construction, jusqu'aux 2 ou 3 ouvriers apostrophés depuis la rue alors qu'ils ravalent la façade d'une habitation dans des conditions de sécurité désastreuses aux yeux de l'inspecteur. Les visites sont inopinées; l'inspecteur se fait accompagner par un représentant de la direction: le patron lui-même ou un ouvrier sur un petit chantier, le chef de chantier, un conducteur de travaux, ou d'autres responsables sur des chantiers plus importants; la visite donne lieu à une lettre d'observation dans laquelle l'inspecteur consigne par écrit ses remarques. Le matériel d'enquête est donc constitué des notes de terrain concernant les visites, des discussions avec les inspecteurs, des documents administratifs (lettres d'observation, procès-verbaux). Ce dispositif d'enquête privilégie bien sûr le point de vue de l'inspecteur, car il inscrit le processus de construction collective des risques dans le cours de son activité professionnelle. Mais il permet aussi, par l'observation des interactions sur les chantiers, et la lecture des documents, de saisir comment sont exprimées, au cours des affaires, les positions des autres acteurs (employeurs, ouvriers) à son égard.

4. Dans son travail sur les «investissements de formes» dans les entreprises, L. Thévenot (1986) met l'accent sur trois caractéristiques des formes: leur degré de stabilité, leur domaine de validité, leur degré d'objectivation.

5. Les CHSCT sont obligatoires pour les établissements de plus de 50 salariés. Ce sont des instances de discussion et de négociation réunissant des délégués représentant les salariés, la direction de l'établissement, l'ingénieur de sécurité, le médecin du travail, ainsi que deux acteurs extérieurs à l'entreprise: l'inspecteur du travail et le contrôleur de la Caisse régionale d'assurance-maladie. 


\section{REPÉRER DES ZONES DE TRAVAIL MOUVANTES}

Au cours de la visite, l'inspecteur traduit le spectacle du chantier en une liste de situations de travail caractérisées par le risque qu'elles comportent. Il note, il enregistre des risques qui iront nourrir le dossier administratif de l'entreprise, détenu au bureau de la section. Une situation de travail comporte à la fois un décor et des personnages occupés dans celui-ci à des activités de travail. Pour argumenter l'existence d'un risque, l'inspecteur doit impérativement rassembler les deux éléments. Il doit montrer que des personnages évoluent, en raison de leur travail, dans le décor qu'il a pointé. En particulier, vis-à-vis du Parquet, le relevé d'une infraction par procèsverbal n'acquiert une quelconque validité que si l'inspecteur apporte la preuve que des ouvriers sont effectivement occupés à la tâche incriminée.

Le chantier offre au regard du visiteur une multitude d'objets et de matériaux, immobiles ou en mouvement, ainsi que des ouvriers qui vont et viennent d'un endroit à un autre, qui commencent une opération, et l'interrompent, qui s'interpellent dans tous les sens. Tout au long de la visite c'est donc une tâche primordiale pour l'inspecteur d'établir quelles sont les zones pertinentes de son intervention. Toute une part des discussions porte sur cette délimitation des territoires travaillés: les employeurs étendent au maximum les zones de no man's land, tandis que l'inspecteur tente de faire la preuve d'une nécessaire présence humaine à l'endroit indiqué. Les voies de passage empruntées épisodiquement par les ouvriers, les zones de stockage de matériaux, tous ces lieux qui, sur un chantier, sont parfois foulés au pied sans l'être constamment, alimentent plus que tout autre ces débats topographiques. À partir des informations qu'il récolte progressivement au cours de la visite sur les zones de travail, l'inspecteur en déduit par un raisonnement logique des voies nécessaires de passage, il interroge des ouvriers, il profite enfin de saisir sur le vif la présence d'un ouvrier, preuve irréfutable, du moins l'espère-t-il, de l'appartenance du lieu à l'ensemble des zones sur lesquelles son intervention est légitime. Parfois, la présence inopinée d'un ouvrier apporte effectivement un démenti cruel aux propos rassurants de l'employeur. Mais la présence, incontestable, d'un ouvrier sur un lieu ne clôt pas toujours la discussion car l'employeur peu dénier à la scène la qualité d'une scène de travail normale et usuelle: l'ouvrier vient ici pour une raison totalement exceptionnelle, il ne respecte pas les consignes de travail, il est ici pour d'autres raisons que son travail.

$\mathrm{Au}$ cours de la visite d'un grand chantier ${ }^{6}$, qui aura duré de 8 h à 12 h 30 , l'inspecteur est accompagné par un conducteur de travaux. Les immeubles en construction de la vaste zone de rénovation en sont à des étapes différentes d'achèvement. Le gros-œuvre est pratiquement fini pour celui qui nous intéresse ici. Nous avons visité un à un les étages et nous accédons enfin à la terrasse supérieure de l'immeuble, par des moyens d'accès sévèrement critiqués par l'inspecteur. Celui-ci fait remarquer qu'une multitude d'objets, coupants et pointus, traînent sur cette terrasse. Il le note sur son carnet.

$E^{7}$ : «Mais personne ne vient plus sur cette terrasse!»

Juste à ce moment-là, nous voyons un ouvrier qui s'affaire sur la terrasse.

I: «Et ce monsieur, vous voulez que je note son nom et que je vérifie s'il existe?»

$\mathrm{E}$ : «C'est moi qui l'ai envoyé à l'instant (suite aux critiques de l'inspecteur sur les moyens d'accès, notre interlocuteur avait demandé à un ouvrier d'aller chercher des planches pour améliorer les accès)!»

I: «Mais alors, si personne ne vient, il faut enlever les moyens d'accès!»

E: «Mais ... On va travailler dessus plus tard.»

I: (avec le sourire): «Alors dans ce cas ... il faudra nettoyer.»

Discuter et enregistrer les risques d'une situation suppose donc acquise pour l'inspecteur la conviction qu'il s'agit bien là d'une zone de travail. Celles-ci évoluant sans cesse, il doit saisir dans l'instant les indices de leur étendue.

Une fois le gros-œuvre achevé, la multitude des entreprises intervenantes (plombiers, peintres, serruriers, électriciens, etc.) est disséminée sur le chantier. Pour déterminer à qui incombe la responsabilité des différents secteurs, l'inspecteur doit donc effectuer un véritable travail cartographique, afin de morceler le chantier en autant de territoires occupés chacun par une entreprise. La question «qui travaille où?» est fondamentale et les différents interlocuteurs ne se privent pas de déplacer

6. Le maître d'œuvre emploie sur le chantier 130 personnes et fait appel à 50 intérimaires.

7. Dans la retranscription des dialogues, nous utilisons les abréviations survantes: E pour «employeur» (ou son représentant), I pour «inspecteur», $C$ pour «contrôleur», $O$ pour «ouvrier». 
la responsabilité de telle opération à d'autres entreprises. Sans responsable, l'inspecteur ne peut pas agir: il perd la menace potentielle du procès-verbal, il ne peut plus rien exiger. L'inspecteur reconstruit les responsabilités à partir d'indices: recueil de paroles et recoupements, repérage du corps de métier (maçons, peintres, ...), couleur des casques et des vêtements de travail.

Le but de ces enquêtes est aussi de détecter toutes les formes d'emplois illégales suspectées sur les chantiers, de découvrir des entreprises non recensées dans les documents officiels (ex: soustraitants de sous-traitants), et qui emploient de plus les ouvriers aux tâches généralement les plus dangereuses et les plus pénibles ${ }^{8}$. Voici un exemple des difficultés rencontrées par les inspecteurs lors des visites pour cartographier les responsabilités:

Le contrôleur visite un chantier pratiquement achevé en ce qui concerne le gros-œuvre. Il ne reste plus que des sous-traitants occupés à la finition: maçonnerie extérieure et intérieure, pose des fenêtres, électricité, ... Nous arrivons dans une zone largement ouverte sur le vide: apparemment les garde-corps ont été enlevés puisque les planches et les piquets métalliques censés les soutenir traînent par terre. Le contrôleur commence à s'énerver en voyant la largeur de l'ouverture non protégée, il trouve ceci inadmissible. Un ouvrier lui répond que c'est parce qu'il y a eu un déchargement de parpaings à l'étage, mais que les garde-corps vont être remis. Le contrôleur cherche à savoir qui travaille dans cette zone: qui a amené les parpaings, quelle entreprise? L'ouvrier lui indique l'entreprise de maçonnerie D.

Nous nous dirigeons vers le chef de l'ouvrier. Le contrôleur lui demande pour qui il travaille. Il ne comprend pas trop ce qu'on lui veut, il vient d'arriver, puis il dit: D. Décidément, le contrôleur va voir le responsable $\mathrm{D}$ qui est de l'autre côté de l'étage. Le contrôleur l'amène sur la zone à problèmes. D dit que ce n'est pas lui qui s'occupe des garde-corps: lui il travaille justement une fois que les garde-corps sont mis et il pose alors ses filets (ses ouvriers font de la maçonnerie sur les murs extérieurs du bâtiment). Le contrôleur: «Je ne cherche pas à savoir qui doit protéger en théorie. À partir du moment où il y a un salarié à vous qui travaille dans cette zone, vous devez assurer sa protection (et il montre le $1^{\text {er }}$ ouvrier que nous avons rencontré et qui travaille apparemment pour D).»

D: «C'est A qui est chargé de mettre les protections. Et la preuve elle est là: nous, nous travaillons après (et il montre un autre ouvrier qui travaillait auparavant dans les parages et qui maintenant installe les garde-corps).» Le contrôleur admet. Mais il fait remarquer que le type devrait avoir un harnais de sécurité.

Les responsabilités sont difficiles à établir lorsque l'inspecteur ne rencontre sur le chantier que l'un des responsables potentiels. Celui-ci a alors beau jeu de déplacer les obligations vers les autres, en leur absence. Le cas de figure courant sur les grands chantiers est celui où le maître d'œuvre accompagne l'inspecteur. Lorsque la situation de travail pose la question de la responsabilité du sous-traitant, ce dernier, occupé sur d'autres chantiers ou au siège de son entreprise, n'a laissé que ses ouvriers à cet endroit, peu compétents ou peu enclins à entrer dans un effort d'éclaircissement des responsabilités. Pour dégager les obligations de chacun, l'inspecteur peut essayer de revenir aux engagements inscrits dans les contrats de sous-traitance, dans les PHS ${ }^{9}$, dans les notices de sécurité que les donneurs d'ordres doivent communiquer aux sous-traitants. Généralement, ces documents apportent des informations bien pauvres au regard des situations de travail observées sur les chantiers. Les entreprises intervenantes ont au fond tout intérêt à entretenir un flou dans leurs rôles respectifs: devant l'administration, chacune peut alors déplacer la responsabilité vers l'autre. Les efforts coûteux de l'inspecteur pour éviter ces ballottements incessants visent avant tout à transformer l'indétermination régnante en frontières nettes. L'inspecteur organise donc des confrontations entre entreprises: sur le chantier, ou lors de réunions spéciales de mise au point. Sa présence contribue alors à créer les conditions nécessaires pour que les différentes entreprises intervenantes, sommées par une autorité extérieure, trouvent entre elles des arrangements relatifs à la prévention des risques. Créer des obligations durables et sans équivoque.

8. Le recours à la sous-traitance et à l'intérim pour les activités les plus dangereuses et une méthode de gestion des risques dans l'entreprise (R. Linhart, 1978; F. Ginsbourger, 1982: et P. Tartakovski, 1985). Le témoignage de G. Wallraff (1986) offre des exemples très instructifs.

9. Le Plan d'hygiène-sécurité est un document qui doit être mis au point avant l'ouverture du chantier, et qui définit par écrit les dispositifs de prévention envisagés pour les différents types d'opérations prévus. Il est obligatoire pour un chantier d'un montant supérieur à 12 millions de francs. Il doit être envoyé à l'inspection du travail (Décret $77-996$ du 19.08.77). 


\section{SAISIR LES ACTIVITÉS DU CHANTIER AU COURS D'UNE VISITE ÉPHÉMÈRE}

Pour lire le chantier, les inspecteurs utilisent volontiers les textes du décret du 8 janvier 1965. Ce sont des textes précis et faciles à manier, ils constituent une grille de lecture puissante pour traduire le spectacle du chantier en une liste de situations non conformes ${ }^{10}$. La traduction du chantier s'applique en premier lieu aux décors de travail. Les articles du décret permettent de lire les objets: existence ou absence d'objets (garde-corps, échelles, ...), caractérisation de ces objets (leur solidité, leur longueur, ...), disposition de ces objets (les bastaings sont-ils jointifs, l'échelle est-elle devant ou derrière les garde-corps?). Le regard, éduqué par les textes, est l'artisan principal de cette lecture. Mais toutes les caractéristiques des objets ne sont pas directement éprouvées par l'œil seul. D'autres épreuves sont nécessaires, qui forgent sur place la réalité de ces objets. La solidité et la stabilité des dispositifs de protection sont testées au cours de mini-épreuves: I'inspecteur appuie sur un garde-corps, secoue le tube d'un échafaudage ou le montant d'une échelle, prend appui. sur le bout d'un bastaing. Les longueurs sont parfois mesurées à l'aide de mètres pliants: hauteur d'un garde-corps, distance entre le mur et l'échafaudage, longueur séparant deux montants d'échafaudages.

Plus que la simple lecture des objets, le spectacle des scènes de travail apporte la preuve des risques inhérents aux modes opératoires des ouvriers. L'inspecteur, cependant, est un visiteur. Le temps de sa présence est infime par rapport à la durée du chantier. Pour construire convenablement les risques du travail, l'inspecteur doit donc être capable de reconstruire, d'après les scènes ponctuelles qu'il observe, l'ensemble des modes opératoires couramment mis en œuvre par les salariés. Ce travail est d'autant plus aisé que les opérations sont standardisées et répétitives. Une seule scène permet alors de comprendre les traits essentiels des cycles de travail sans cesse répétés. Sur les chantiers les modes opératoires sont peu standardisés et certaines scènes dangereuses sont de plus très ponctuelles.

Les ouvriers et leur encadrement sont engagés dans des ajustements continuels visant à adapter les matériaux, les équipements, et les principes de travail aux situations particulières. Par conséquent, l'inspecteur se trouve lui-même confronté à la difficulté d'imaginer, à partir de ce qui lui est donné à voir, l'ensemble des cas de figure, et donc des risques, auxquels sont exposés les ouvriers. Les indices des risques, recueillis au cours de la visite, sont alors bien pauvres.

Lorsque les déclarations d'ouvertures de chantier ne sont pas envoyées à l'inspection du travail, lorsque le chantier dure peu de temps, lorsqu'il est situé dans des lieux difficilement visibles de la voie publique, les traces de son existence deviennent elles-mêmes raréfiées. Les employeurs tirent parti de cette discrétion pour prendre des libertés avec la réglementation. La chasse aux. risques se rapproche alors de la chasse au travail clandestin: toute une investigation est nécessaire aux inspecteurs pour découvrir les lieux d'activité.

Sur une petite route de campagne, le contrôleur aperçoit incidemment, depuis sa voiture, une maison dont la toiture est en train d'être refaite. Une rangée d'arbres la cache en partie. Il y a une personne sur le toit, et une autre qui lui envoie les tuiles depuis le sol. Il n'y a aucune protection apparente. Le contrôleur s'arrête et se dirige vers la maison. Après une discussion un peu tendue avec le patron, qui passait les tuiles à son jeune apprenti perché sur le toit:

C: Sur des toitures comme ça, vous ne mettez pas de protection!

E: Franchement, pour vous répondre, on en met à la ville, mais ici on est bien, on est tranquille ... alors on n'en a pas mis.

\section{DES RISQUES PONCTUELS, DES COÛTS DE PRÉVENTION DISPROPORTIONNÉS}

Une fois identifiées les zones de travail et les pratiques des ouvriers sur le chantier, inspecteurs et employeurs discutent de la réalité des risques encourus. Les premiers montrent l'ampleur des dangers afin d'argumenter le bien-fondé de leurs exigences, tandis que les deuxièmes minimisent ces dangers afin de démontrer le bon état de leur chantier. Au cours de ces échanges, des employeurs tirent parti du caractère évolutif des situations de travail: quand bien même l'inspecteur aurait démontré l'existence d'un risque, son interlocuteur lui montrera que le travail est si ponctuel qu'il y a une chance infime pour que l'accident survienne. Le coût d'un dispositif de sécurité serait

10. On trouvera en annexe des exemples d'articles de ce décret. 
totalement disproportionné au risque global occasionné par la courte période de travail nécessaire. L'inspecteur pointe-t-il des risques de chute pour des serruriers qui posent sans protection une rampe d'escalier? L'opération ne dure pas plus de 10 minutes. Peut-on raisonnablement dans ces conditions, demande l'employeur, imposer une journée de travail pour installer puis démonter un échafaudage?

L'inspecteur peut bien sûr couper court à l'argumentation et imposer sans convaincre, utiliser la menace d'un procès-verbal. Mais il ne peut pas recourir systématiquement à cette arme coûteuse devant chaque situation à risque: il n'en a pas le temps; et le Parquet en classerait une bonne partie sans suite. Il doit donc rester vigilant toutes les fois qu'il use de la menace ou de la notification du procès verbal, s'il veut garder sa crédibilité. Il doit si possible garder intact le lien établi entre le relevé de l'infraction et la peine, entre les menaces proférées et leur mise à exécution. L'entrée de l'inspecteur sur le chantier vise à recomposer «l'économie des intérêts» des employeurs, pour reprendre l'expression de M. Foucault (1975). L'art de punir des inspecteurs, comme celui élaboré par les réformateurs à l'avènement du Code pénal, est basé sur une «technologie de la représentation». L'inspecteur doit maîtriser la représentation que l'employeur se fait de la portée de ses sanctions. Contraint par les suites données par le Parquet et les juges à ses sanctions, l'inspecteur doit prudemment manier ses armes vis-à-vis des employeurs. La fugacité des chantiers offre des points de résistances aux employeurs. Soit l'inspecteur cède devant la ponctualité des risques, soit il est acculé aux menaces de sanction.

Indépendamment du niveau de risque, les déplacements incessants sur les chantiers augmentent les coûts de la sécurité. Ils abolissent la notion de poste conçu comme un lieu fixe équipé pour une longue période. Sur un poste fixe il est possible d'installer une fois pour toutes un dispositif de sécurité. Sur un chantier, il faut réinstaller à chaque fois de nouveaux dispositifs.

Des électriciens sont juchés sur les montants les plus hauts d'un échafaudage roulant, afin de pouvoir atteindre le plafond sur lequel ils installent des câbles, à environ $7 \mathrm{~m}$ de haut. Le contrôleur signale les risques importants de chute.

L'un des électriciens: «on est habitué à travailler comme ça, on a pas de problème».

Au contrôleur qui lui fait remarquer qu'il faudrait au moins s'attacher avec une corde, il réplique qu'une corde ce n'est pas possible à mettre, parce que l'échafaudage est tout le temps déplacé d'un endroit à un autre.

Le contrôleur s'incline.

Le déplacement des dispositifs de sécurité est alors d'autant plus difficile que la configuration des postes successifs de travail est peu standardisée. Un faible niveau de standardisation nécessite non seulement de déplacer, mais aussi d'adapter constamment les principes de sécurité aux nouvelles situations. La difficulté est donc d'autant plus grande que le bâtiment est pétri d'angles, de coins, de façades orientées dans tous les sens. Autant les grandes lignes, les angles droits, la régularité des dispositions, rendent possibles la répétition des mesures de sécurité, leur simple déplacement, autant la rupture architecturale de ces régularités nécessite l'intervention continuelle de nouveaux assemblages. La puissance de prévision en est réduite d'autant, le PHS ne définit plus que les grandes lignes, la sécurité du chantier est une improvisation continuelle. Comme le dit un responsable sur un chantier constitué d'innombrables immeubles relativement biscornus, lorsque l'inspecteur lui reproche de n'avoir pas su prévoir les problèmes: «vous savez il y a 60 cas différents, c'est jamais identique!»

La mise en place des dispositifs préventifs requiert la participation des ouvriers. Que ce soit pour l'installation systématique de garde-corps sur les plates-formes de travail, pour l'aménagement de platelages corrects, ou pour l'utilisation des moyens de protection individuels, les ouvriers sont un point de passage obligé entre les demandes de l'inspecteur et le chantier. Au nom de la sécurité, les inspecteurs demandent aux employeurs, le cas échéant, de renforcer la surveillance sur les ouvriers et d'exercer leur pouvoir disciplinaire. C'est sans doute l'un des moments de l'action d'inspection où la référence primordiale à la sécurité apparaît le plus clairement puisqu'elle s'impose face à des principes très fortement mis en avant par ailleurs comme la défense des salariés.

Sur un petit chantier, trois salariés sont occupés avec leur patron à la couverture d'une maison. Il n'y a pas de protection collective (échafaudage sur les côtés), ni de protection individuelle (harnais). La discussion s'est engagée depuis quelques instants déjà. Le patron dit finalement qu'il est d'accord pour installer un échafaudage. «Mais le problème aussi, c'est que pour la sécurité les gars ils suivent pas toujours bien non plus!» Le contrôleur: «Là-dessus moi je vais vous dire ce que je dis à chaque fois. Les gars ils veulent pas appliquer les consignes? Un avertissement. Ça recommence? Vous pouvez les mettre à pied ou les 
licencier. Je vous assure, si un salarié vient se plaindre chez moi et me dit «mon patron m'a licencié parce que je ne respectais pas les consignes de sécurité», je lui dis «Monsieur, votre patron il a eu raison de vous licencier!»

Sur les chantiers, le contrôle minutieux et constant des activités des ouvriers est difficile. L'instauration d'une discipline semblable à celle des grands ateliers des manufactures ou des chaînes de montage est ici impossible. Les ouvriers se déplacent sans cesse, nul poste de surveillance ne confè̀e aux conduites une visibilité constante. Ce n'est pas, comme dans les grands ateliers de confection, le règne de la transparence et de l'immobilité, mais celui de l'opacité et des mouvements continuels. Le travail n'est pas non plus standardisé. Les gestes des ouvriers ne sont pas fractionnés en autant de cellules minutieusement prescrites. Les techniques de contrôle à distance, par le rythme de la machine ou par la mesure précise des temps, sont ici inopérantes. Les employeurs composent avec cette irréductible autonomie des ouvriers du bâtiment ${ }^{11}$. Face aux inspecteurs, ils tirent parti de cette situation pour tout à la fois déplacer la responsabilité du risque vers les ouvriers et écarter leur propre responsabilité hiérarchique, du fait des difficultés incompressibles de surveillance. «On ne pelıt pas toujours être derrière leur dos!» avancent-ils sans cesse. L'inspecteur est là dans une position difficile: soit il fait activement pression pour que l'employeur instaure un contrôle des conduites plus strict en matière de sécurité, mais il risque ainsi, en créant des «gendarmes de la sécurité», de voir utiliser celle-ci comme prétexte à un exercice renforcé et abusif à ses yeux du pouvoir disciplinaire, et de plus il instaure un clivage profond entre les ouvriers du chantier et luimême; soit il n'insiste pas, et ses arguments sur la surveillance prennent, aux yeux de l'employeur, un caractère purement tactique au sein de la discussion, sans conséquences pour l'avenir.

\section{VISITES ET CONTRE-VISITES}

Après chaque visite l'inspecteur envoie une lettre d'observation à l'employeur, où il reprend par écrit les positions qu'il avait énoncées lors de la visite. Ensuite se pose la question du contrôle par l'inspecteur de l'évolution du chantier: quelle réponse l'employeur a-t-il finalement donnée aux demandes de l'inspecteur? A-t-il respecté ses engagements oraux? Les réponses orales ou mêmes écrites de l'employeur sont une étape. L'épreuve de force ultime entre l'inspecteur et l'employeur est réalisée sur le chantier, par le constat de ce qu'a fait l'employeur.

Obstacle massif à l'action de l'inspecteur au cours de la visite, la fugacité des chantiers l'est autant pour le suivi de l'entreprise. Lors des visites ultérieures, l'inspecteur ne lit plus seulement le chantier à partir du code du travail, mais aussi à partir de ses propres remarques et des engagements de l'employeur, élaborés auparavant. Il doit alors habilement évaluer la période adéquate qui sépare la visite de la contrevisite. En effet si la période est trop courte, l'employeur n'aura pas eu, légitimement, le temps de faire appliquer les mesures de sécurité nécessaires. Par contre, si la période est trop longue, le chantier aura tellement progressé que l'inspecteur ne retrouvera plus les mêmes situations. Le contrôle du respect des engagements n'aura plus de support, les objets du contrôle ayant disparu.

Les inspecteurs remédient alors au caractère fugitif des chantiers par la construction des similitudes entre les situations. C'est pour eux une question d'efficacité et d'économie. Une question d'économie car une remarque faite pour telle situation singulière sera valable pour toutes les situations similaires: l'employeur devra donc s'y conformer à chaque fois. Une question d'efficacité car c'est la seule façon pour eux de juger, lors des contre-visites, le chantier à l'aune des engagements pris lors de la visite précédente. C'est par une telle construction des similitudes que l'inspecteur peut utiliser au cours des visites la mémoire des visites précédentes, malgré l'évolution du chantier. Il ne repart pas chaque fois à zéro. L'inspecteur peut alors s'engager dans une gradation des menaces qui vont peser sur l'employeur. Même si la situation, stricto sensu, n'est pas la même que la dernière fois, il pourra faire référence aux engagements pris par l'employeur sur des situations de ce type. Autour des similitudes, les inspecteurs et les employeurs ont alors des positions opposées: les premiers tenteront de les construire, les deuxièmes de les briser.

Sur ce chantier l'inspecteur a notifié un procès-verbal pour l'absence de garde-corps aux échafaudages et l'absence de moyens d'accès sûrs. Il revient la semaine suivante. Avec le responsable il retourne aux lieux visés la dernière fois. Tout est en ordre. Il lui rappelle que

11. La taylorisation des chantiers se heurte à la conception traditionnelle des «métiers du bâtiment». Pour une description de la «langue de métier» chez les tailleurs de pierre, voir D. Cru (1985). 
selon lui il est inadmissible que des règles «élémentaires» de sécurité n'aient pas été respectées sur le chantier, et il maintient le procès-verbal malgré les améliorations apportées depuis. L'employeur dit qu'effectivement, le lendemain même des observations, il avait remédié aux défauts. On passe devant le bâtiment d'à côté. L'inspecteur remarque les échafaudages du premier étage. Il constate qu'à d'autres endroits, nouvellement investis par les ouvriers, il n'y a toujours pas de garde-corps.

I: Regardez ça ce laissez-aller, de qui se fout-on? On se fout du monde sur ce chantier. Je ne parlerai pas de la stabilité, OK admettons. Mais vous voyez les planches en travers qui servent de garde-corps! Je vous rappelle qu'un garde-corps c'est une lisse, une souslisse et une plinthe.

E: C'est parce que l'échafaudage est en construction. Il l'a laissé comme ça à 5 heures et puis il est parti.

I: Oui .... (sceptique)

De retour aux baraquements:

I: Bon vous voyez ce que je vous ai dit.

E: rappelle qu'ils ont fait le nécessaire le lendemain des observations. L'inspecteur le reconnaît. «Mais ça ne va pas s'il faut qu'on soit tout le temps derrière vous. Une remarque faite ici est valable pour l'ensemble du chantier et pour l'avenir.»

E: «Mais un chantier c'est évolutif. C'est comme ça un jour et un autre c'est autrement. Ça change, on peut pas être toujours derrière les gars.»

I: «Moi ma politique c'est d'établir une relation de confiance avec les entreprises. Mais pour cela il ne faut pas avoir à répéter à chaque fois la même chose!»

À la fin de la visite, l'employeur essaye de faire revenir l'inspecteur sur sa décision (le PV). Celui-ci ne cède pas: «je ne peux pas laisser passer ça!»

La fugacité des chantiers tend à isoler chaque visite, elle brise le développement d'une action graduée de l'inspecteur sur les employeurs du bâtiment. Ce faisant, elle évite aussi aux inspecteurs des épreuves de force délicates. En effet, engagés dans des confrontations directes avec les employeurs au cours des visites, les inspecteurs sont parfois amenés, avant toute chose, à sauver la face (voir E. Goffman, 1974). C'est essentiellement le cas lorsque des risques importants sont constatés, mais que la discussion s'achemine vers un constat explicite d'absence de solution. Pour montrer combien la sécurité les imprègne, pour ne pas avouer la défaite de la sécurité devant les contraintes du chantier, ils proposent alors, dans la discussion, des mesures théoriquement efficaces, mais dont eux-mêmes savent qu'elles seront très vraisemblablement inappliquées. Le harnais de sécurité est un très bon outil de ce genre: un «joker» de l'argumentation. Le harnais est le repli idéal des inspecteurs lorsqu'ils admettent les arguments des employeurs sur le coût des protections collectives. Mais la faiblesse du lien entre le harnais et le corps des ouvriers est tel que tous les acteurs savent que son usage est de fait extrêment rare.

L'inspecteur effectue une première visite sur un grand chantier de construction d'immeubles. Le maitre d'œuvre emploie 85 personnes en période de pointe, 40 actuellement. Accompagné par le chef de chantier $\mathrm{H}$, l'inspecteur regarde un ouvrier en équilibre sur des poutres, occupé à une tâche de coffrage. Tous reconnaissent la difficulté d'installer des protections collectives en phase de coffrage.

I: «Dans ce cas, il faut travailler avec un harnais.»

$\mathrm{H}$ n'est pas d'accord car, pour ancrer le harnais quelque part, il faut garder trop de longe, ce qui fait que le harnais devient totalement inutile en cas de chute. H s'impatiente et dit que de toute façon il est impossible d'être en permanence derrière les «bonshommes». L'inspecteur calme le jeu. Il n'en pense pas moins que le harnais pourrait être utilisé pour éviter de basculer de l'autre côté du muret.

$\mathrm{R}$, un conducteur de travaux, intervient pour signaler qu'il est toujours difficile de protéger le bord des tables de coffrage, même si celles-ci sont de conception moderne.

$\mathrm{I}$ : «Si une protection collective est impossible, alors il faut utiliser le harnais.»

Silence des deux côtés. R sourit avec un air moqueur.

I (mi-sérieux): «Vous avez l'air sceptique Mr R.»

$\mathrm{R}$ : «Vous le savez bien Mr A (l'inspecteur), les gens ne mettront jamais le harnais!»

I: «... Ben oui. Pour parler franc, oui. Mais, dans la mesure du possible...»

$H$ : «Il faudrait un haut-parleur pour répéter constamment les consignes!»

La connivence tacite sur l'impossibilité d'imposer le harnais, brisée ici par l'humour de l'inspecteur et du responsable de chantier, me paraît au fondement de nombre d'accords sur les chantiers. L'inspecteur garde la façade en proposant le harnais. L'employeur tombe d'accord avec 
lui. Un compromis est donc trouvé où chacun exprime son action pour la sécurité ${ }^{12}$. La fugacité des chantiers devient alors une aide pour l'inspecteur. Engagé dans le face à face, il s'est risqué à formuler des exigences irréalistes. À la visite suivante, le temps aura de toute façon enfoui la situation à risque. Nulle constatation ne pourra plus venir apporter de démenti éclatant à l'efficacité de ses demandes. Réciproquement, l'employeur du bâtiment peut, plus qu'ailleurs, s'engager à prendre toutes les mesures nécessaires. Il tablera sur la faible probabilité d'un contrôle de l'inspecteur avant que l'évolution du chantier ne rende méconnaissables les zones de travail. La fugacité du chantier tend à séparer, pour chacun des acteurs, l'instant du face à face des contrôles ultérieurs. Elle facilite alors, dans les échanges de paroles ou de lettres, une expression partagée du souci de la sécurité, dissociée de sa mise en œuvre sur le chantier.

\section{L'ABSENCE DE RELAIS}

Les chantiers ne sont pas les seuls lieux de travail caractérisés par leur instabilité. Dans certains établissements industriels les inspecteurs du travail se heurtent aux variations incessantes des équipements, des produits, des techniques. Ils éprouvent des difficultés à reconstruire les risques à partir des visites, à discerner les équipements réellement utilisés, à exiger des investissements importants pour des risques ponctuels, à imposer la mise en place systématique de dispositifs de sécurité sur des configurations changeantes. Cependant, dans les établissements dotés d'institutions représentatives du personnel (délégués du personnel, mais surtout CHSCT), de services de médecine du travail, les inspecteurs disposent dans l'entreprise de relais présents plus régulièrement qu'eux dans les ateliers. Ces relais vont effectuer à leur place tout le travail de traduction de l'usine en une série de risques. Ils seront là pour discuter régulièrement avec la direction des dispositifs de prévention.

Ces relais internes à l'entreprise construisent les fils nécessaires de la continuité qui manquent si cruellement aux inspecteurs en visite sur les chantiers. Car dans le bâtiment ces relais sont pratiquement inexistants. L'action de l'inspecteur est une confrontation directe avec la direction des entreprises ou ses représentants. Bien sûr l'inspecteur en visite pose des questions aux ouvriers et aux. chefs pour recueillir des informations complémentaires aux indices directement visibles sur le chantier. L'inspecteur interroge l'encadrement, depuis le chef de chantier jusqu'aux chefs d'équipe, pour comprendre les détails et les fondements techniques des opérations dont les scènes de travail observées ne permettent pas de saisir l'intégralité, ou pour reconstituer une opération qu'aucune scène de travail, par malchance, ne vient illustrer pendant la visite. Il interroge les ouvriers pour recueillir des renseignements complémentaires, notamment dans le domaine des protections individuelles: chaussures, casques, lunettes, gants, etc. Est-ce que l'employeur fournit des moyens de protection? en quelle quantité? Sont-elles adaptées? Est-ce que les ouvriers les portent? Mais, étranger au chantier, l'inspecteur est dans la position d'un ethnographe qui disposerait de mauvais informateurs.

Soucieux de limiter les motifs de litige avec l'inspection du travail, l'employeur présentera des noodes opératoires conformes aux règles de sécurité. Ignorant eux-mêmes, sur les grands chantiers, les pratiques usuelles en vigueur sur le terrain, les responsables présenteront uniquement les principes opératoires consignés par écrit dans les documents prévisionnels. Soucieux d'éviter les problèmes ultérieurs avec leurs supérieurs hiérarchiques, les ouvriers hésiteront à parler des pratiques non conformes aux consignes internes de sécurité ou à la réglementation du travail. De plus, appréciant mal, selon les inspecteurs, les risques «réels» et se préoccupant peu de sécurité, ils seront tenus pour de piètres informateurs. Quel que soit le poste occupé par son interlocuteur, l'inspecteur dispose donc très rarement sur les chantiers de relais lui apportant, au-delà d'informations élémentaires, un concours fiable au repérage des risques. Il ne dispose ni des cadres institutionnels stables (CHSCT) qui lui permettraient de recueillir les informations importantes sur la sécurité; ni du temps nécessaire, du fait du caractère temporaire des chantiers, pour tisser avec les différents acteurs de l'entreprise ces réseaux de liens personnels qui créent les conditions propices à la constitution de bons informateurs.

Cette absence de relais a des conséquences primordiales sur la pratique des inspecteurs. Elle donne à la visite, à ce parcours systématique des lieux du chantier, un statut privilégié dans

12. M. Juffe (1980) n'hésite pas, quant à lui, à dénoncer les institutions de prévention des risques professionnels comme participant à une entreprise collective d'occultation des accidents du travail. 
l'élaboration des prises de position sur les risques et les mesures préventives. Le regard qui suit attentivement le spectacle du chantier devient l'outil principal d'objectivation des risques. La compétence de l'inspecteur passe par l'éducation de son regard, qui devra saisir les indices significatifs de l'état du chantier. L'inspecteur met en æuvre un regard clinique sur les chantiers, de la même façon qu'un médecin recourt à une clinique pour examiner le corps du malade. La visite de chantier est un examen. Sur le chantier, constamment à la recherche des traces de ce qu'il ne peut voir directement dans l'instant, comme visiteur de passage, l'inspecteur construit la réalité selon un paradigme indiciel (C. Ginzburg 1980). Comme le détective, comme le psychanalyste, l'inspecteur travaille sur des indices. Des indices produits dans son cas par le regard, plutôt que par le recueil de paroles; des indices qui permettent de surprendre quelques fragments de la vie du chantier; des indices qui mettent sur la piste de risques, mais qu'il faudra traduire en termes juridiques et qu'il faudra constituer en preuves, si l'inspecteur, par la suite, veut convaincre le Parquet de la nécessité des poursuites, et les juges de celle des peines.

Dans ce travail du regard, les chantiers du bâtiment présentent alors deux faces pour l'inspecteur. D'un côté une facilité pour son action de prévention: des risques dont le repérage est aisé. Les chantiers exigent en effet, pour une première approche, peu de compétence technique (il suffit essentiellement de connaître les termes du métier pour désigner les équipements); ils exigent peu de compétence juridique: les textes sont précis et aisés à manier, ils nécessitent donc peu de recherches pour traduire les situations à risque en termes juridiques; l'application du regard aux objets et aux scènes de travail suffit pour définir une liste conséquente de risques. D'un autre côté le bâtiment offre, en raison de sa fugacité, des obstacles importants à l'inspecteur, dès qu'il s'engage plus avant dans les contrôles de sécurité. Saisir une chronologie du chantier à partir d'une vision nécessairement éphémère; dire rapidement, dans l'instant, les risques de situations prêtes à se transformer; établir malgré tout des fils entre les visites successives sur un chantier qui n'est déjà plus le même: pour relever ces défis du bâtiment, l'inspecteur ne dispose pas, sur les chantiers, des relais nécessaires. Bien que ce secteur soit, en raison d'indices statistiques alarmants, une cible centrale pour une action de prévention, les coûts qu'il multiplie pour le travail des inspecteurs pourraient détourner ceux-ci vers des terrains plus faciles.

ANNEXE

Décret du 18 janvier $1965\left(n^{\circ} 65-48\right)$

\footnotetext{
portant sur «les mesures particulières de protection et de salubrité applicables aux établissements dont le personnel exécute des travaux de bâtiment, des travaux publics, et tous autres travaux concernant les immeubles».
}

— Ce décret comporte 16 titres: I Mesures générales de sécurité, II Appareils de levage, III Câbles, chaînes, cordages et crochets, IV Travaux de terrassement à ciel ouvert, V Travaux souterrains, VI Travaux de démolition, VII Échafaudages, plates-formes, passerelles et escaliers, VIII Échelles en bois, IX Travaux sur les toitures. X Travaux de montage, de démontage et de levage de charpentes et ossatures, XI Travaux de construction comportant la mise en ceuvre d'éléments préfabriqués lourds, XII Travaux au voisinage de lignes, canalisations et installations électriques, XIII Mesures générales d'hygiène, XIV Logement provisoire des travailleurs, XV Dispositions particulières, XVl Dispositions finales.

Au total, 235 articles.

- Nous citerons deux exemples d'articles couramment cités par les inspecteurs l'un très précis:

ART. 115: «Les échafaudages doivent être munis, sur les côtés extérieurs,

$1^{\circ}$ de garde-corps constitués par deux lisses placées l'une à un mètre, l'autre à $45 \mathrm{~cm}$ au-dessus du plancher;

$2^{\circ}$ de plinthes d'une hauteur de $15 \mathrm{~cm}$ au moins.

Toutefois, ces prescriptions ne font pas obstacle à l'établissement de dispositifs de protection d'une efficacité au moins équivalente.»

L'autre beaucoup plus général:

ART. 116, al. 1: «Dans le cas où la protection collective du personnel ne peut être aş̧rée d'une manière satisfaisante, des appareils, équipements ou produits protecteurs appropriés (tels que des ceintures ou baudriers de sécurité, casques, lunettes, bottes, vêtements imperméables, gants, brassières, maniques, épaulières, tabliers, enduits aptes à s'opposer à l'action du ciment) doivent être mis à la disposition des travailleurs.» 


\section{RÉFÉRENCES BIBLIOGRAPHIQUES}

CRU, D., «Les tailleurs de pierre», Travail. $\mathrm{n}^{\circ} 7,1985$, pp. 48-55.

DODIER, N., l'Inspection du travail face à la diversité des entreprises, Rapport de recherche, Centre d'études de l'Emploi, Paris, 1986.

FOUCAULT, M., Surveiller et punir, naissance de la prison, Paris, Gallimard, 1975.

GINSEOURGER, F., «La «répa» à Marseille, Crise et statut de la classe ouvrière», Travail, n 1, 1983, pp. 19-27.

GINZEURG, C., «Signes, traces, pistes. Racines d'un paradigme de l'indice», le Débat, n 6, 1980, pp. 4-44.

GOFFMAN, 'E., les Rites d'interaction, Paris, Minuit, 1974.

JUFFÉ, M., À corps perdu, ou l'accident du travail existe-t-il?, Paris, Seuil, 1980.

LINHART, R., «Procès de travail et division de la classe ouvrière», dans la Division du travail, Colloque de Dourdan, Paris, éditions Galilée, 1978.

TARTAKOSKI, P., les Intérimeurs, Paris, Messidor, 1985.

THÉVENOT, L., «Les investissements de forme», Cahiers du Centre d'études de l'emploi, $\mathrm{n}^{\circ}$ spécial Conventions économiques, 1986, pp. 21-72.

WALLRAFF, G., Tête de Turc, Paris, La Découverte, 1986.

\section{RÉSUMMÉ}

Le secteur du bâtiment est caractérisé en France par un taux d'accidents du travail particulièrement élevé. À partir d'une série d'observations de type ethnographique, l'auteur analyse les visites des inspecteurs du travail sur les chantiers comme des processus de construction sociale des risques et comme des négociations autour des dispositifs de sécurité. L'exemple du bâtiment met en évidence l'influence du degré de stabilité des formes d'organisation du travail sur la mise en ouvre d'une politique de prévention des risques professionnels. L'auteur montre en effet les multiples difficultés que la forte instabilité des chantiers crée pour les interventions des inspecteurs, tant dans le repérage des risques que dans le contrôle de l'installation effective des mesures de protection.

\section{SUMMARY}

The construction sector in France is characterized by a particularly high rate of occupational accidents. From a series of ethnographic type observations, the author analyzes the visits of work inspectors to construction sites as a process of the social construction of risks and as negotiations relating to safety measures. The construction example demonstrates the influence of the degree of stability of forms of work organization on the application of a policy for the prevention of occupational risks. The author shows the many difficulties that the severe instability of construction sites creates for interventions by inspectors, both in the identification of risks and in the control of the effective installation of protective measures.

\section{RESUMEN}

El sector de la construcción se ha caracterizado en Francia por una tasa de accidentes de trabajo particularmente elevada. A partir de una serie de observaciones de tipo etnográfico, el autor analiza las visitas de los inspectores del trabajo a la obra como proceso de construcción social de riesgos y como negociaciones concernientes a los dispositivos de seguridad. El ejemplo de la construcción pone en evidencia la influencia del grado de estabilidad de las formas de organización del trabajo sobre la puesta en práctica de una política de prevención de los riesgos profesionales. El autor muestra en efecto las múltiples dificultades que la gran inestabilidad de las obras, crea para las intervenciones de los inspectores, tanto en la localización de los riesgos como en el control de la instalación efectiva de medidas de protección. 